The surprising result, however, is that the expected chemical linkage between the polymerized units is not $3^{\prime}-5^{\prime}$ (as it is in RNA and DNA), but turned out to be principally a mixture of $2^{\prime}-5^{\prime}$ and $5^{\prime}-5^{\prime}$. Whether the linkage formed is determined by the use of carbodiimide is uncertain, but the results certainly suggest that prebiotic nucleic acids may not have been pure $3^{\prime}-5^{\prime}$ linked polynucleotides. Perhaps it is an accident of history that the first enzyme which copied nucleic acids made $3^{\prime}-5^{\prime}$ linkages. Or did DNA, in which only $3^{\prime}-5^{\prime}$ (and not $2^{\prime}-5^{\prime}$ ) linkages are possible, precede RNA ?

\section{Light on Sweetness}

\section{from our Molecular Biology Correspondent}

TASTE is a subject which, despite its obvious interest, is still so obscure that it even comes as a surprise to find it represented in the biochemical literature. Although a certain amount is known about the physical response of the taste receptors-in particular that the primary process can be described in terms of a simple adsorption isotherm defined by a single equilibrium constant-little systematic effort seems hitherto to have been invested in attempts to isolate active receptor molecules.

Rather more than a year ago, the isolation of a sweet-sensitive molecule-a protein-from bovine taste buds was reported by Dastoli and Price, and its further characterization is now described (Dastoli et al., Biochemistry, 17, 1160; 1968). The assay for receptor activity consists in measurement of the binding constants for sugars by determining refractive index increments. (The observed increments are surprisingly large, and the stated reproducibility is high; a spectrophotometric method is also mentioned in the earlier paper, but details are not given.) Over a wide $p H$ range, the binding remains essentially constant, but falls off below $p \mathrm{H} 6$. The most striking result is that the binding constants follow the order of sweetness of the sugars, as measured in vivo (behavioural assay). Saccharine also gives a high binding constant. The $p \mathrm{H}$ dependence of the binding follows the in vivo sweetness response.

The new results include characterization of the protein in a number of respects; it has been obtained as an essentially homogeneous component, with a molecular weight of 150,000 . Amino-acid analysis shows it to be rather basic. The article is succinct to the point of being telegraphic, and more details would be of interest. Measurements of binding constants as a function of temperature will be important in order to tell whether earlier predictions about the entropic character of the binding are borne out.

\section{RNA Phages}

\section{from our Cell Biology Correspondent}

THe properties of a new RNA bacteriophage R23, discovered recently in August's laboratory at the Albert Einstein College of Medicine, New York, have now been described by Watanabe, Watanabe and August (J. Mol. Biol., 33, 1; 1968). R23 is chemically, physically and immunologically similar to $f 2$, but it has the remarkable property of dominating RNA synthesis in the $E$. coli host cells. Following infection with $R 23, E$. coli RNA synthesis is markedly inhibited and newly synthesized RNA is both qualitatively and quantitatively phage specific. In this respect $R 23$ is more like the $T$ even phages than $f 2$ which, according to Zinder, has little effect on host RNA metabolism.

After infection with $R 23,60$ per cent of the RNA made is encapsulated into phage particles whereas with $f 2$ and $Q \beta$ only 25-30 per cent of the RNA is encapsulated. Moreover, the other $30-40$ per cent of the RNA made during $R 23$ infection is RNase resistant and therefore phage specific. $R 23$ is thus unique among the RNA phages because it completely inhibits host RNA synthesis. How the inhibition is achieved is unknown. Synthesis of bacterial protein is also inhibited by $R 23$. Initially $R 23$ specifies an RNA polymerase, but later in infection coat protein synthesis predominates and late in infection almost all newly made protein is phage coat. Coat protein synthesis, as expected, was not detectable in the absence of phage RNA synthesis. It is now clear that ability of RNA phages to switch off host RNA synthesis varies from $f 2, M S 2$ and $Q \beta$ at one extreme, which have little effect, through $R 17$ and $\beta$ which have a limited inhibitory effect to $R 23$ which inhibits completely.

In a subsequent paper (ibid., 21) Watanabe and August report that the antibiotic and anti-tumour agent phleomycin inhibits the replication of $R 23$ just as it inhibits bacterial RNA metabolism and DNA phage replication. Addition of phleomycin to $E$. coli infected with $R 23$ simultaneously blocks synthesis of double and single stranded phage RNA molecules. In vitro phleomycin inhibits both DNA and RNA dependent RNA synthesis and, as judged from thermal melting curves, the antibiotic binds to both RNA and DNA. It does not, however, block translation of $m$ RNA.

The nucleotide sequence at the $5^{\prime}$ terminus of another RNA phage, this time $M S 2$, has recently been reported by Glitz (Biochemistry, \%, 927; 1968). Using MS2 RNA uniformly labelled with ${ }^{32} \mathrm{P}$, alkaline hydrolysis and DEAE-cellulose column chromatography-virtually identical procedures to those used by Roblin during his work on the $5^{\prime}$ terminus of $R 17$ (see Nature, 217,606 ; 1968)—Glitz has shown that the $5^{\prime}$ terminus of $M S 2$ is pppGpGpU. It appears that $R 17$ and $M S 2$ have identical $5^{\prime}$ termini.

Another particularly interesting experiment with the RNA from the phage $R 17$ has recently been described by Spahr and Gesteland (Proc. US Nat. Acad. Sci., 59, 876; 1968). They have cleaved the $R 17$ RNA molecule into two pieces, one sedimenting at about $15 S$ and the other at $21 S$, with a new endonuclease, RNase IV isolated from $E$. coli MRE-600. The $15 S$ piece has the 5 terminus of the intact molecule because on hydrolysis it liberates the tetraphosphate pppGp. In preliminary experiments, with a cell-free system and polyacrylamide gel electrophoresis of the products, they find that the $15 S$ piece specifies coat protein, albeit at low efficiency, whereas the larger fragment specifies $R 17$ RNA synthetase. This result confirms that the coat protein cistron is the first. It also suggests that the most likely order of the cistron is coat, A protein, and last RNA synthetase. Furthermore, it proves each cistron can be translated independently of the others. In some basically similar experiments,'Lodish (J. Mol. Biol., 32, 681 ; 1968) has reached the same conclusion. 